\title{
The Robustness of Graph $k$-shell Structure under Adversarial Attacks
}

\author{
Bo Zhou, Yuqian Lv, Yongchao Mao, Jinhuan Wang, Shanqing Yu, and Qi Xuan, Member, IEEE
}

\begin{abstract}
The $\boldsymbol{k}$-shell decomposition plays an important role in unveiling the structural properties of a network, i.e., it is widely adopted to find the densest part of a network across a broad range of scientific fields, including Internet, biological networks, social networks etc. However, there arises concern about the robustness of the $k$-shell structure when networks suffer from adversarial attacks. Here, we introduce and formalize the problem of $k$ shell attack and develop an efficient strategy to attack the $k$-shell structure by rewiring a small number of links. To the best of our knowledge, it is the first time to study the robustness of graph $k$-shell structure under adversarial attacks. In particular, we propose a Simulated Annealing (SA) based $k$-shell attack method and testify it on four real-world social networks. The extensive experiments validate that the $k$-shell structure of a network is robust under random perturbation, but it is quite vulnerable under adversarial attack, e.g., in Dolphin and Throne networks, more than $40 \%$ nodes change their $k$-shell values when only $10 \%$ links are changed based on our SA-based $k$-shell attack. Such results suggest that a single structural feature could also be significantly disturbed when only a small fraction of links are changed purposefully in a network. Therefore, it could be an interesting topic to improve the robustness of various network properties against adversarial attack in the future.
\end{abstract}

Index Terms-Social network, $k$-shell decomposition, adversarial attack, simulated annealing, structural feature, random perturbation.

\section{INTRODUCTION}

$\mathbf{N}$ ETWORKS or graphs can well represent various complex systems in our daily life [1], such as social networks [2], biological networks [3], power networks [4], and financial networks [5]. Network science has been a very active field nowadays, due to its highly interdisciplinary attributes, and most of the tasks in this domain are related to network structure, such as link prediction [6], node classification [7], network reconstruction [8]. Considering the simplicity and broad applicability, $k$-shell decomposition, which aims to find the largest subgraph of a network by recursively removing the nodes with degree less than $k$, i.e., each node has $k$ neighbors in the remaining graph at least [9], has become one of the most widely used methods to describe network structure. For instance, Altaf et al. [10] proposed a procedure to predict the feature of functional-unknown proteins based

This work was supported in part by the National Natural Science Foundation of China under Grants 61973273, by the Zhejiang Provincial Natural Science Foundation of China under Grants LR19F030001, and by the Research and Development Center of Transport Industry of New Generation of Artificial Intelligence Technology. (Corresponding authors: Qi Xuan.)

B. Zhou, Y. Lv, Y. Mao, J. Wang, S. Yu, and Q. Xuan are with the Institute of Cyberspace Security, College of Information Engineering, Zhejiang University of Technology, Hangzhou 310023, China (e-mail: xuanqi@zjut.edu.cn).

B. Zhou is also with Zhejiang Institute of Communications, Hangzhou 311112, China.

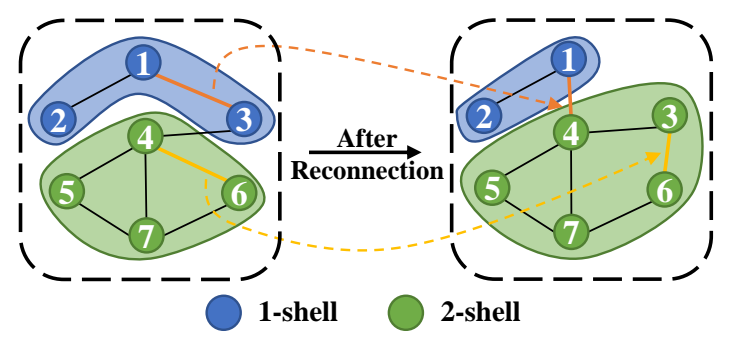

Fig. 1. Node 3 is from 1-shell to 2 -shell after rewiring two links, i.e., changing $e_{13}$ and $e_{46}$ to $e_{14}$ and $e_{36}$.

upon $k$-shell. Gaertler et al. [11] found $k$-shell is useful in examining the Autonomous System (AS) Graph. Many researchers have proposed a number of methods [12]-[17] based on $k$-shell decomposition to identify the key nodes in a network or to measure the influence of users in online social networks. Miorandi et al. [18] extended the $k$-shell decomposition method to determine the impact of nodes on spreading epidemics in dynamic complex networks.

With the rapid development of $k$-shell applications in various fields, it would be an interesting question to ask: how robust the graph $k$-shell structure is under various perturbations? Indeed, Kitsak et al. [19] found that $k$-shell decomposition is robust under random deletions of even up to $50 \%$ of the links. However, there is still a lack of study on the robustness of the $k$-shell structure under adversarial attack or purpose perturbation. For example, as illustrated in Fig. 1, originally node 3 belongs to 1 -shell; after rewiring two links, it turns to be 2-shell immediately. From a realistic point of view, in a credit scoring system, fraudsters can establish fake connections with several high-credit customers to evade the $k$-shell decomposition to disturb the scoring system; and spammers can create fake followers easily to improve its centrality online, so as to increase the chance of fake news being spread to others. It is thus urgent to investigate the robustness of graph $k$-shell structure under various adversarial attacks.

Quite recently, numerous researchers devote themselves into the study of adversarial attacks on networks. Zügner et al. [20] proposed a method to attack graph convolutional networks (GCN) for node classification. Yu et al. [21] proposed three heuristic strategies by rewiring to attacked the RA link prediction method successfully. Chen et al. [22] proposed a Genetic Algorithm (GA) based Q-Attack to destroy the network community structure. Xuan et al. [23] disturbed the degree distribution of networks to test the robustness of Broido and Clauset (BC) classification for scale-free networks in terms of statistical measures. Nonetheless, as far as we know, 


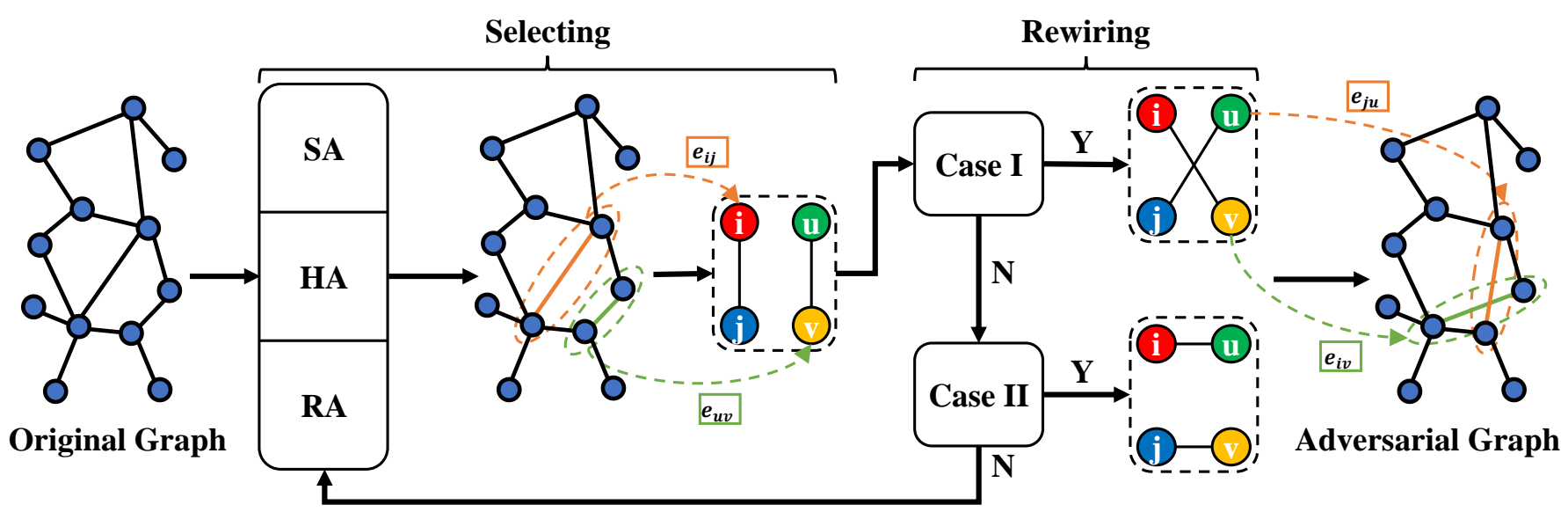

Fig. 2. The overall framework of $k$-shell attack. In selection module, two links $e_{i j}$ and $e_{u v}$ are selected. In rewiring module, if the two selected links satisfy Case I condition, we perform rewiring operation and the two links become $e_{i v}$ and $e_{j u}$, obtaining the adversarial graph.

most of these studies focus on destroying machine learning algorithms on graphs but rarely concern the robustness of a particular feature, such as $k$-shell, which is the basis of network analysis and graph machine algorithms in fact. We thus argue that it may be more crucial to investigate the robustness of particular structural features under adversarial attacks, which could provide deeper insight to understand the robustness of network structure from various aspects.

In this paper, we propose the robustness of graph $k$-shell structure under adversarial attacks for the first time, and make the following contributions:

1) We study the adversarial attack on $k$-shell decomposition by rewiring links, for the first time.

2) We adopt the Simulated Annealing (SA) optimization method to select candidate links for rewiring, so as to realize the effective $k$-shell attack.

3) We validate that the $k$-shell structure of real-world networks are indeed much more vulnerable to our SA-based $k$-shell attack, by comparing with Random rewiring Attack (RA) and Heuristic rewiring Attack (HA).

The rest of paper is organized as follows. In Section II we introduce our methodology, including basic ideas, evaluation metrics, and our SA-based $k$-shell attack. In Section III the experimental results on four real-world networks are presented to validate the effectiveness of our attack method. Finally, the paper is concluded in Section IV.

\section{Methodology}

In this part, we will introduce our methods to testify the robustness of graph $k$-shell structure.

\section{A. Preliminaries and Ideas}

Given an undirected graph $G=(V, E)$ without self-loop, where $V$ is the set of nodes and $E \subseteq(V \times V)$ is the set of links, each node $v_{i}$ is assigned an index $k_{i}$ to represent its $k$ shell value based on the $k$-shell decomposition as a recursively pruning process. Nodes with the same value of $k$ constitute the $k$-shell. And all the nodes with their indices not smaller than $k$ constitute $k$-core. Each node has its own $k$-core and $k$-shell. The graph under adversarial attack is denoted as $G^{\prime}=\left(V^{\prime}, E^{\prime}\right)$, where $V^{\prime}=V$ and the $k$-shell of node $v_{i}$ under adversarial attack is denoted as $k_{i}^{\prime}$.

The whole attack process is illustrated in Fig. 2, where Case I and Case II are stated as below:

- Case I: If link $e_{i v} \notin E$ and $e_{j u} \notin E$, meanwhile, $v_{i}$ and $v_{j}$ are different from $v_{v}$ and $v_{u}$, respectively.

- Case II: If link $e_{i u} \notin E$ and $e_{j v} \notin E$, meanwhile, $v_{i}$ and $v_{j}$ are different from $v_{u}$ and $v_{v}$, respectively;

Then, the attack is realized by iteratively implementing the following two steps:

1) Selecting: We select a pair of links, i.e., $e_{i j}$ and $e_{u v}$, from the original graph through particular methods, including Random, Heuristic and SA-based $k$-shell attack, which will be introduced in detail in Section II-C.

2) Rewiring: We first release $e_{i j}$ and $e_{u v}$, and then establish two new links $e_{i v}$ and $e_{j u}$ if Case $\mathbf{I}$ is satisfied but Case II is not; otherwise, establish $e_{i u}$ and $e_{j v}$ if Case II is satisfied but Case I is not.

Note that our rewiring will not change the degree of nodes, making the attacks imperceptible to certain extent [24], [25].

\section{B. Evaluation Metrics}

We use the Attack Success Rate (ASR), Link Change Rate (LCR), and Link Per Node (LPN) to measure the effectiveness of various attacks.

- ASR: If the $k$-shell of node $v_{i}$ is successfully changed after the attack, i.e., $k_{i} \neq k_{i}^{\prime}$, we set $r_{i}=1$, otherwise $r_{i}=0$, then ASR is defined as:

$$
\operatorname{ASR}=\frac{\sum_{v_{i} \in V} r_{i}}{|V|},
$$

where $|V|$ denotes the total number of nodes in $G$. The larger the ASR is, the more successful the attack is.

- LCR: If the link $e_{i j}$ is changed after the attack, we set $\psi_{i j}=1$, otherwise $\psi_{i j}=0$. then LCR is defined as:

$$
\mathrm{LCR}=\frac{\sum_{e_{i j} \in E} \psi_{i j}}{|E|},
$$

where $|E|$ denotes the total number of links in $G$. LCR denotes the budget of attack, the smaller of which, the less noticeable of the attack method. 
- LPN: In most cases, it would be interesting to ask that how many links do we need to change to successfully attack a node on average. We thus define LPN as follows:

$$
\mathrm{LPN}=\frac{\sum_{e_{i j} \in E} \psi_{i j}}{\sum_{v_{i} \in V} r_{i}} .
$$

Similarly, the smaller of LPN, the higher effectiveness of the attack method.

\section{SA-based k-shell Attack}

Simulated Annealing (SA) is an optimization method, which has been used in many research areas [26]. Our SA-based $k$ shell attack is constituted of attacking iteration and annealing iteration, where the latter is nested within the former.

Attacking: In the attacking iteration at round $n$, we put the adversarial graph $G_{n-1}^{\prime}$ into annealing iteration (simply set $G_{0}^{\prime}=G$ ) and get $G_{n}^{\prime}$ as the output. The above attacking process will continue $N$ times, and output the final adversarial graph $G_{\text {out }}=G_{N}^{\prime}$.

Annealing: The initial and terminate temperature of the annealing are set to $T$ and $T_{m}$, respectively. $T_{\tau}$ represents the temperature of the system in the annealing iteration at round $\tau$, with $T_{0}=T$. At round $\tau$, the current temperature $T_{\tau}$ is updated to:

$$
T_{\tau}=\frac{T_{\tau-1}}{\tau} .
$$

We then randomly select a pair of links satisfying Case I or Case II, as introduced in Section [II-A] and rewire them on $G_{n-1}^{\prime}$ to get an adversarial graph denoted as $G_{a}$. After that, we can calculate $\mathrm{ASR}_{\tau}$ based on $G_{a}$ at this round by Eq. (1). Note that, here we simply set $\mathrm{ASR}_{0}$ as the attack success rate of $G_{n-1}^{\prime}$. If $\mathrm{ASR}_{\tau}>\mathrm{ASR}_{\tau-1}$, this rewiring operation will be accepted and recorded directly; otherwise, it will be accepted with the probability:

$$
p_{\tau}=e^{-\left|\mathrm{ASR}_{\tau}-\mathrm{ASR}_{\tau-1}\right| / T_{\tau}} .
$$

This process will not be terminated until $T_{\tau} \leq T_{m}$. Finally, we set $G_{n}^{\prime}=G_{a}$ and trigger the next round of attacking.

\section{EXPERIMENTS}

In this section, we compare our SA-based $k$-shell attack with Random rewiring Attack (RA) and Heuristic rewiring Attack (HA), where RA refers to selecting links completely at random and HA refers to purposefully selecting two links that one is linked with nodes of high $k$-shell and the other is linked with nodes of low $k$-shell. We will realize the attacks based on the two-step rewiring mechanism introduced in Section [II-A and testify them on four different real-world networks.

\section{A. Datasets}

The four real-world networks are briefly introduced in the following, with their basic properties presented in Table I]

- Karate: It is a social network, which describes the interactions among the members in a university karate recorded by Zachary [27].

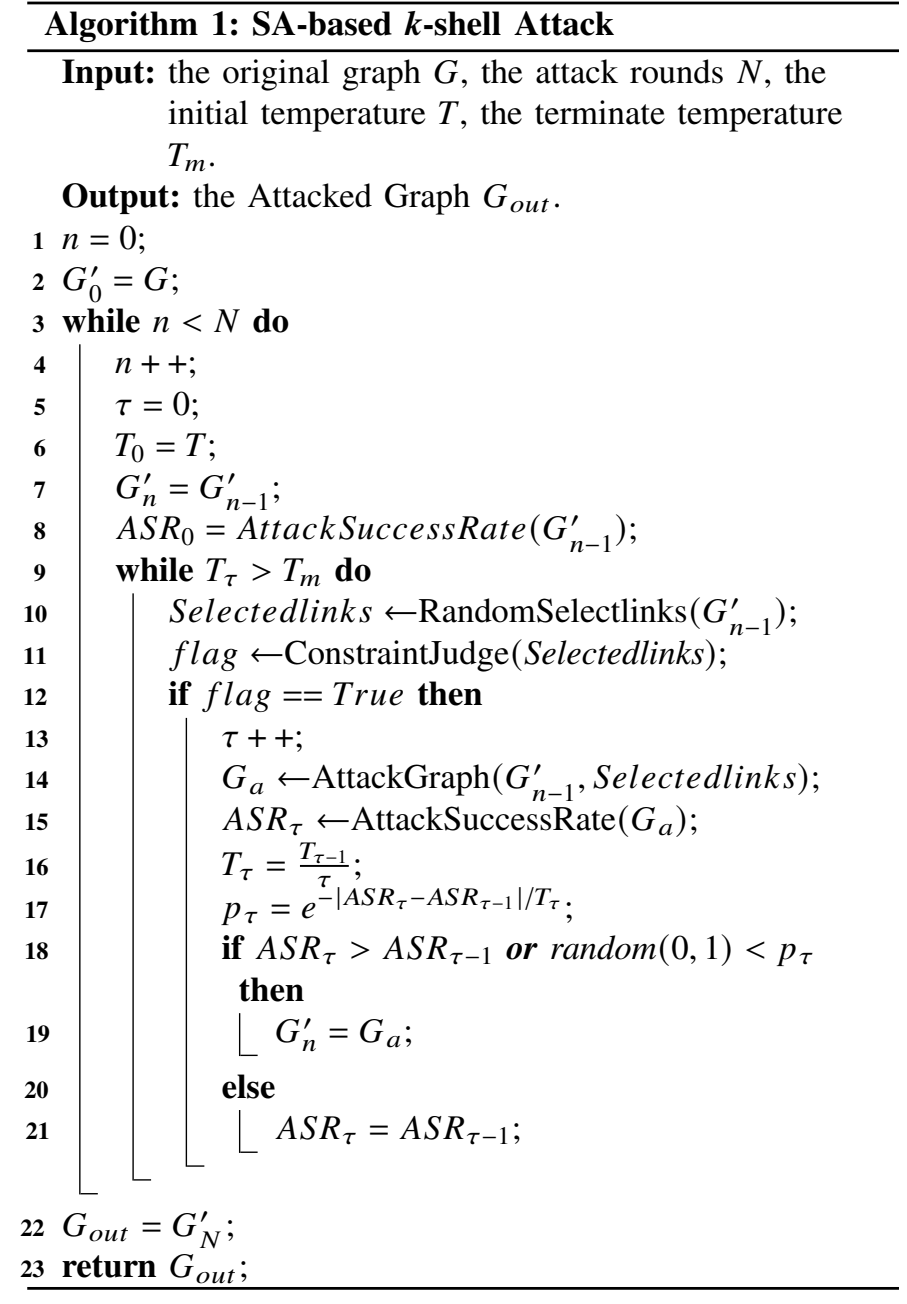

- Dolphin: It is a social network that describes the frequent associations between bottlenose dolphins living off Doubtful Sound, New Zealand [28].

- Thrones: It is a network of characters in an American fantasy drama television series, Game of Thrones [29].

- Facebook: It is a online social network of students at the University of California, Irvine, which includes the users that sent or received at least one message [30].

TABLE I

STATISTICS OF FOUR REAL-WORLD NETWORKS

\begin{tabular}{cccc}
\hline Dataset & \#Nodes & \#Links & Maximum $k$-shell \\
\hline Karate & 34 & 78 & 4 \\
Dolphin & 62 & 159 & 4 \\
Thrones & 107 & 352 & 7 \\
Facebook & 1266 & 6451 & 11 \\
\hline
\end{tabular}

\section{B. Results and Analysis}

Next, we focus on ASR and LPN as the functions of LCR on the four networks, as shown in Fig. 3, based on which we can get the following results.

Firstly, indeed, the $k$-shell structure of real-world networks are much more vulnerable to adversarial attacks, compared with RA. As indicated by Kitsak et al. [19], k-shell decomposition is quite robust under random perturbation, e.g., in 


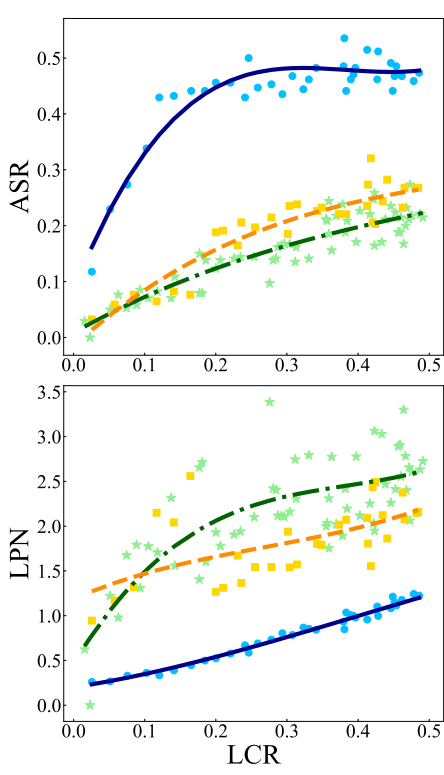

(a) Karate
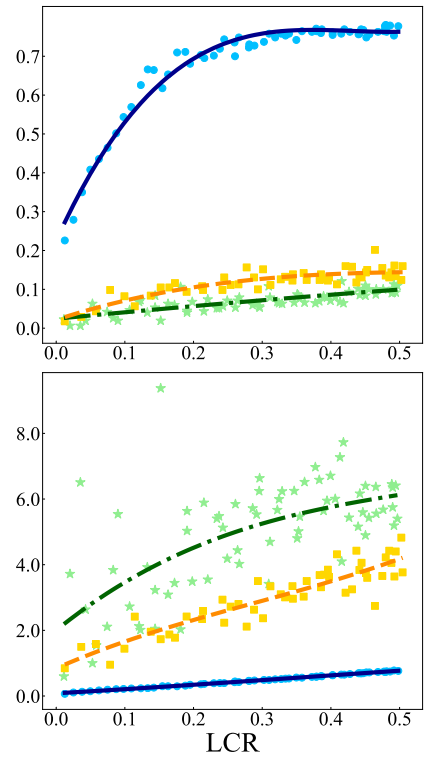

(b) Dolphin
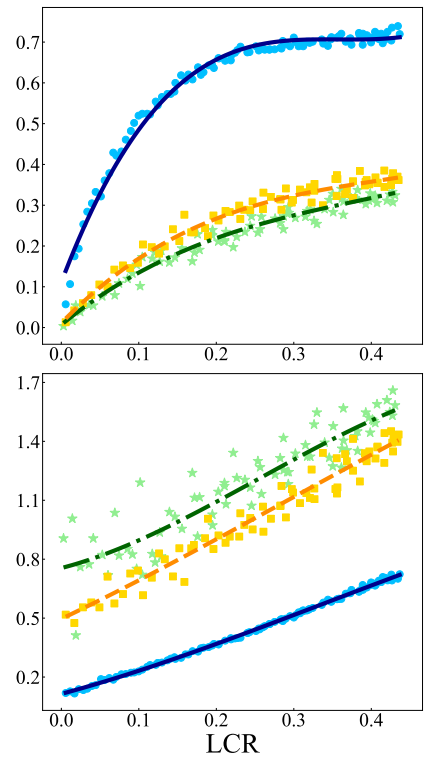

(c) Thrones
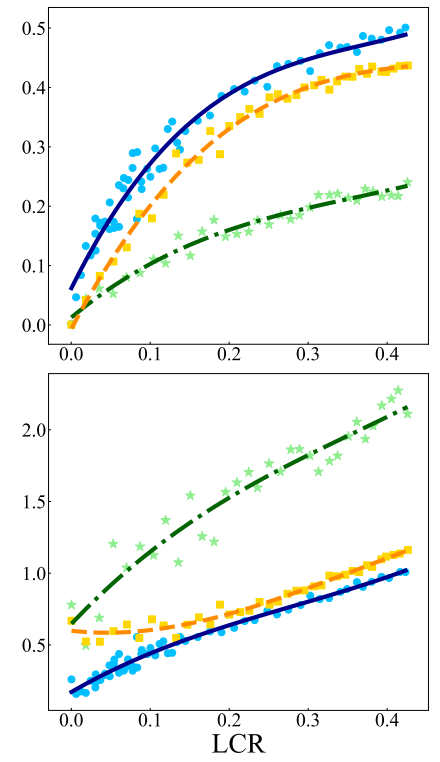

(d) Facebook

experimental results of $\mathrm{SA}$ - fitting line of SA

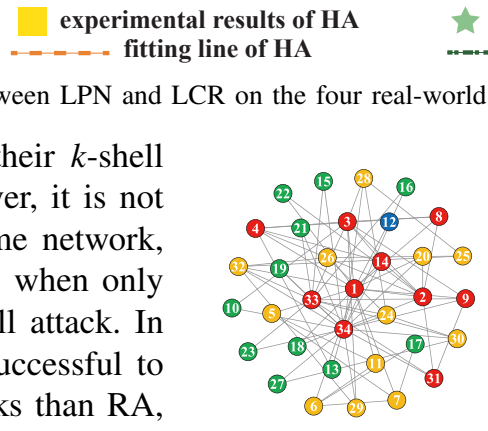

(a) Original Graph

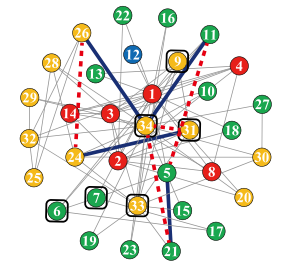

(b) $\mathrm{LCR}=0.051$

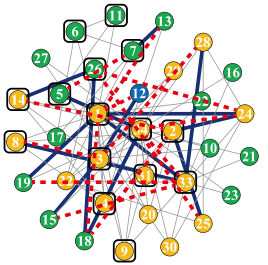

(c) $\mathrm{LCR}=0.205$

Secondly, SA-based $k$-shell attack is also much more effective than RA and HA. To successfully change the $k$-shell value of a node, only less than one link is needed to change in most cases, even when to realize the large-scale attack (corresponding to large LCR). But for RA, it may need to change more than four links to successfully attack one node in Dolphin network. Again, HA falls in between the two. Surprisingly, HA behaves quite well on attacking Facebook network, with relatively high ASR but low LPN. We found that, compared with the other three networks, a larger fraction of nodes in Facebook network have extremely large or small $k$-shell values, i.e., $18 \%$ of nodes have 11 -shell and $19 \%$ of nodes have 1-shell. Since HA tends to decrease those high $k$ shell values but increase those low ones, it matches Facebook network quite well and thus can efficiently disturb the $k$-shell values of the nodes in this network.

As a case study, we visualize the rewired links on Karate network under the SA-based $k$-shell attack with different LCR, as shown in Fig. 4 It can be seen that $k$-shell attack is a typical combination optimization problem, i.e., different LCR values correspond to different groups of rewiring links. For instance, link $e_{26,34}$ is added to the original graph when LCR $=0.05$ while it is not when LCR $=0.205$. Meanwhile, we find that the $k$-shell of node $v_{26}$ changes from 3 to 2 as the LCR changes from 0.051 to 0.205 , while none of the links connected to it

\section{1-shell
original link
2-shell}

Fig. 4. (a) The original graph. (b) and (c) represent the adversarial graphs obtained by SA-based $k$-shell attack under $\mathrm{LCR}=0.051$ and $\mathrm{LCR}=0.205$, respectively, on Karate network.

change in this process. This phenomenon suggests that we can change the $k$-shell value of a target node without changing the links connected to it, making the attack quite imperceptible.

\section{CONCLUSION}

This paper has investigated the robustness of $k$-shell structure under adversarial attacks for the first time, and has demonstrated that $k$-shell decomposition is vulnerable to adversarial attacks. That is, a large fraction of nodes in a network will change their $k$-shell values by only rewiring a relatively small ratio of links, using our SA-based $k$-shell attack method. This phenomenon is quite different from the finding of Kitsak et al. [19] that $k$-shell decomposition is robust under random deletions of even up to $50 \%$ of the links.

Note that here we only focus on changing the $k$-shell values of as many nodes as possible and the SA-based attack method is rather time-consuming. But in reality, it may be more crucial to increase or decrease the $k$-shell values of a small number 
of target nodes more efficiently, which could be a good topic to study in the future.

\section{REFERENCES}

[1] Q. Xuan, Z. Ruan, and Y. Min, Graph Data Mining: Algorithm, Security and Application. Springer, 2021.

[2] A. W. Wolfe, "Social network analysis: Methods and applications," Contemporary Sociology, vol. 91, no. 435, pp. 219-220, 1995.

[3] B. H. Junker and F. Schreiber, Analysis of biological networks. John Wiley \& Sons, 2011, vol. 2.

[4] G. Paul, T. Tanizawa, S. Havlin, and H. E. Stanley, "Optimization of robustness of complex networks," The European Physical Journal B, vol. 38, no. 2, pp. 187-191, 2004.

[5] S. Bougheas and A. P. Kirman, Complex Financial Networks and Systemic Risk: A Review. CESifo, 2014.

[6] C. Fu, M. Zhao, L. Fan, X. Chen, J. Chen, Z. Wu, Y. Xia, and Q. Xuan, "Link weight prediction using supervised learning methods and its application to yelp layered network," IEEE Transactions on Knowledge and Data Engineering, vol. 30, no. 8, pp. 1507-1518, 2018.

[7] S. Dabhi and M. Parmar, "Nodenet: A graph regularised neural network for node classification," arXiv preprint arXiv:2006.09022, 2020.

[8] M. J. Herrgård, N. Swainston, P. Dobson, W. B. Dunn, K. Y. Arga, M. Arvas, N. Blüthgen, S. Borger, R. Costenoble, M. Heinemann et al., "A consensus yeast metabolic network reconstruction obtained from a community approach to systems biology," Nature biotechnology, vol. 26, no. 10 , pp. $1155-1160,2008$

[9] Y. X. Kong, G. Y. Shi, R. J. Wu, and Y. C. Zhang, "k -core: Theories and applications," Physics Reports, vol. 832, pp. 1-32, 2019.

[10] M. Altaf-Ul-Amine, K. Nishikata, T. Korna, T. Miyasato, Y. Shinbo, M. Arifuzzaman, C. Wada, M. Maeda, T. Oshima, H. Mori et al., "Prediction of protein functions based on k-cores of protein-protein interaction networks and amino acid sequences," Genome Informatics, vol. 14, pp. 498-499, 2003

[11] M. Gaertler, "Dynamic analysis of the autonomous system graph," in IPS 2004, International Workshop on Inter-domain Performance and Simulation, 2004.

[12] M. Boguñá, R. Pastor-Satorras, A. Díaz-Guilera, and A. Arenas, "Models of social networks based on social distance attachment," Phys. Rev. E, vol. 70, p. 056122, Nov 2004. [Online]. Available: https://link.aps.org/doi/10.1103/PhysRevE.70.056122

[13] M. Cha, H. Haddadi, F. Benevenuto, and K. Gummadi, "Measuring user influence in twitter: The million follower fallacy," in Proceedings of the international AAAI conference on web and social media, vol. 4, no. 1, 2010.

[14] C. Castellano and R. Pastor-Satorras, "Thresholds for epidemic spreading in networks," Physical Review Letters, vol. 105, no. 21, p. 218701, 2010.

[22] J. Chen, L. Chen, Y. Chen, M. Zhao, S. Yu, Q. Xuan, and X. Yang, "Ga-based q-attack on community detection," IEEE Transactions on Computational Social Systems, vol. 6, no. 3, pp. 491-503, 2019.
[15] G. Bruno, P. Nicola, V. Alessandro, and P. Matjaz, "Modeling users' activity on twitter networks: Validation of dunbar's number," PLOS ONE, vol. 6, 2011.

[16] M. A. Al-garadi, K. D. Varathan, and S. D. Ravana, "Identification of influential spreaders in online social networks using interaction weighted k-core decomposition method," Physica A: Statistical Mechanics and its Applications, vol. 468, pp. 278-288, 2017.

[17] J. Wang, P. Chen, B. Ma, J. Zhou, Z. Ruan, G. Chen, and Q. Xuan, "Sampling subgraph network with application to graph classification," arXiv preprint arXiv:2102.05272, 2021.

[18] A. Garas, F. Schweitzer, and S. Havlin, "Ak-shell decomposition method for weighted networks," New Journal of Physics, vol. 14, no. 8, p. 083030, aug 2012. [Online]. Available: https://doi.org/10. 1088/1367-2630/14/8/083030

[19] M. Kitsak, L. K. Gallos, S. Havlin, F. Liljeros, L. Muchnik, H. E. Stanley, and H. A. Makse, "Identification of influential spreaders in complex networks," Nature physics, vol. 6, no. 11, pp. 888-893, 2010.

[20] D. Zügner, A. Akbarnejad, and S. Günnemann, "Adversarial attacks on neural networks for graph data," in Proceedings of the 24th ACM SIGKDD International Conference on Knowledge Discovery \& Data Mining, 2018, pp. 2847-2856.

[21] S. Yu, M. Zhao, C. Fu, J. Zheng, H. Huang, X. Shu, Q. Xuan, and G. Chen, "Target defense against link-prediction-based attacks via evolutionary perturbations," IEEE Transactions on Knowledge and Data Engineering, vol. 33, no. 2, pp. 754-767, 2021.

[23] Q. Xuan, Y. Shan, J. Wang, Z. Ruan, and G. Chen, "Adversarial attack on bc classification for scale-free networks," Chaos: An Interdisciplinary Journal of Nonlinear Science, vol. 30, no. 8, p. 083102, 2020.

[24] C. M. Schneider, A. A. Moreira, J. S. Andrade, S. Havlin, and H. J. Herrmann, "Mitigation of malicious attacks on networks," Proceedings of the National Academy of Sciences, vol. 108, no. 10, pp. 3838-3841, 2011.

[25] A. Zeng and W. Liu, "Enhancing network robustness against malicious attacks," Physical Review E, vol. 85, no. 6, p. 066130, 2012

[26] J. Wu and Y. Xia, "Complex-network-inspired design of traffic generation patterns in communication networks," IEEE Transactions on Circuits and Systems II: Express Briefs, vol. 64, no. 5, pp. 590-594, 2016.

[27] W. W. Zachary, "An information flow model for conflict and fission in small groups," Journal of anthropological research, vol. 33, no. 4, pp. 452-473, 1977 .

[28] D. Lusseau, K. Schneider, O. J. Boisseau, P. Haase, E. Slooten, and S. M. Dawson, "The bottlenose dolphin community of doubtful sound features a large proportion of long-lasting associations," Behavioral Ecology and Sociobiology, vol. 54, no. 4, pp. 396-405, 2003.

[29] A. Beveridge and J. Shan, "Network of thrones," Math Horizons, vol. 23, no. 4, pp. 18-22, 2016.

[30] T. Opsahl and P. Panzarasa, "Clustering in weighted networks," Social networks, vol. 31, no. 2, pp. 155-163, 2009. 